\title{
PD-L2 Peptide-Montanide Vaccine
}

National Cancer Institute

\section{Source}

National Cancer Institute. PD-L2 Peptide-Montanide Vaccine. NCI Thesaurus. Code C154563.

A vaccine composed of a peptide derived from the immune checkpoint molecule programmed death lig and 2 (PD-L2) combined with the immunoadjuvant montanide ISA51, with potential immunomodulating and antineoplastic activities. Vaccination with PDL2 peptide-montanide vaccine may mount an immune response against PD-L2 expressing cells. This may enhance T-cell proliferation, cytokine production, and T-cell mediated cytolysis. PD-L2 binding to its cognate receptor, programmed cell death protein 1 (PD-1; PDCD1; CD279), downregulates T-cell responses and enhances immune evasion. Montanide ISA-51, also known as incomplete Freund's adjuvant or IFA, is a stabilized water-in-oil emulsion adjuvant containing mineral oil with mannide oleate added as a surfactant that non-specifically stimulates cell-mediated immune responses to antigens. 